\title{
Studying the effects of traits in the genotype of three maize hybrids in Hungary
}

\author{
Seyed Mohammad Nasir Mousavi - Karina Bianka Bodnár - János Nagy \\ University of Debrecen Faculty of Agricultural and Food Sciences and Environmental Management, \\ Institute of Land Use,Technology and Regional Development \\ nasir@agr.unideb.hu
}

\begin{abstract}
SUMMARY
In this study, we selected three hybrids (Armagnac, Loupiac, and Sushi) for evaluation of the effect of traits in genotype in Debrecen. In 2017, the total rainfall from May to October was $314 \mathrm{~mm}$ in Debrecen, which was $236 \mathrm{~mm}$ in the winter period before sowing. The obtained results showed that there was a positive correlation between the weight of the cob maize and the rate of seed/cob, number of rows with number of seeds in column and outer diameter ear with weight of cob and number of rows in grain per ear and the rate of seed / cob; Also, there was a negative correlation between grain weight in ear with seed/cob rate, outer diameter ear and the rate of seed/cob and outer diameter ear with the number of leaves. There was a positive correlation between stem diameter, Seed/cob rate and the number of nodes by GGE biplot. In addition, there are traits of weight of all seeds and outer ear diameter that had the highest effect on average yield. Moreover, the number of seeds per row showed the least effect on the average yield of hybrids.
\end{abstract}

Keywords: Maize, Correlation, GGE biplot

\section{INTRODUCTION}

Maize consumption is divided into three types of human feed, livestock, poultry and industrial uses. Maize grain is very important because of its energy supply for livestock and poultry, and it is used extensively for fattening and it is consumed in most countries, amounting to about $80 \%$ of maize crops. In the industry, maize grain is also used. Hydrolysis of maize starch can produce a variety of food products such as starch, maize, syrup and dextrose. Dry maize starch for food is used in washing shops and for other industrial purposes (Chockan, 2012). Maize is a key candidate for ethanol production, as it yields maize grain which is converted to ethanol (Schwietzke, 2009).

As regards the importance of using biplot, the interaction of genotype with traits (GT) can be used for comparing cultivars based on the difference in traits and identifying cultivars in some desirable traits. For this reason, a candidate for a parent in a corrective program can be used. The biplot of the polygon allows the GT to analyze the pattern obtained to identify the desired genotypes, which is the best solution for some traits. The vector of traits allows analyzing relationships between traits as well as independent selection criteria based on different traits (Yan and Rajcan, 2002).

Knowing the stages of maize growth and development is very important. The length of the life of the maize crop varies from planting to physiological reach and its subsequent stages, between genotypes and different groups, and is influenced by genetic and environmental factors. The most important environmental factors that affect maize phenology include temperature and irradiance (Chogan, 2010).

The GGE biplot has been used to identify high yield and adapted cultivars by many researchers such as Fan et al. (2007) and Setimela et al. (2007) for maize, Yan et al. (2000) and Morris et al. (2004) for wheat, Samonte et al. (2005) for rice, Dehghani et al. (2006) and Yan and Tinker (2007) for barley, Sabaghnia et al. (2006) for lentils and Kang et al. (2006) for common bean. Furthermore, superior crop cultivars must be evaluated on the basis of multiple traits to ensure that the selected cultivars have acceptable performance in variable environments within the target region (Yan and Rajcan, 2002; Yan and Tinker, 2007).

Beiragi et al., 2011 in a research report stated that there is a positive or negative correlation between the measured traits in the correlation coefficient between yield and yield components. These results indicated the complexity of the relationship between grain yield components. The results of the polygonal pattern of genotypes in comparison with adjectives in three years showed that 700-KSC genotype had more value for the number of rows in grain and grain diameter. 602-OSSK and 684 ZP had a higher value for the weight of 1000 seeds and the number of seeds per row. As a result, biplot GT inter relates traits and identifies hybrids that describe desirable traits (Beiragi et al., 2011).

\section{MATERIAL AND METHODS}

Samples were taken in Debrecen. The Experimental Station is situated on the Hajdúság Loess Ridge, and the soil is lowland chernozem with lime deposits and a deep humus layer, formed on loess. It has medium hard loam texture. The soil type is solonetz, strongly calcareous, meadow chernozem, with loam or sandy loam texture.

The sum of daily precipitation was determined by local measurements, while the daily radiation and temperature data were provided by the Meteorological Observatory Debrecen the National Meteorological Service in Budapest. In 2017 the total rainfall from May until October was $314 \mathrm{~mm}$ in Debrecen, which was 236 $\mathrm{mm}$ in winter period prior to sowing.

In Hungary, the daily variation of temperature from 10 to $12^{\circ} \mathrm{C}$ is almost constant from the aspect of the phenological phases of maize; based on these findings, 
this is also the case in another region. Detailed investigations were performed in Canada, USA, and in Western and Eastern Europe. Extended climatological analyses revealed that, in the region of $40-46^{\circ}$ latitude of North America, the daily variation of the temperature during the vegetative phase of vegetative development of maize (May-July) was around $12{ }^{\circ} \mathrm{C}$, and the difference between the regions referred to was some tenth of degrees only, whereas in Western Europe - e.g. England, where maize is grown - daily variation is 6.5 ${ }^{\circ} \mathrm{C}$. In Western Europe the variation is $10.0-11.0^{\circ} \mathrm{C}$, in Southern Europe $6.0-8.0{ }^{\circ} \mathrm{C}$, after all, the question of base temperature should be solved by different approaches in those regions.

We measured important traits in maize, including the number of rows (NR), number seeds on per cob (NSC), Weight of all seeds (WS), weight of cob (WC), seed/cob rate (SC), Number of seeds in column (NOSC), height plant (HP), Length ear (LE), outer ear diameter (OED), number of nodes (NON), stem diameter (SD), leaf number (LN), weight 1000 seeds in fresh (WSW) and hybrids in this experiment were Armagnac FAO 490, Loupiac FAO 380, Sushi FAO 340. We analyzed this data with the Gen Stat software that has powerful spreadsheet facilities for data storage and manipulation and an attractive graphics viewer that allows users to edit and interact with their plots.

\section{RESULTS}

\section{Evaluation of Pearson's linear correlation coefficients between traits}

The correlation results in this experiment indicate that there is a positive correlation between the weight of the cob maize and the rate of seed/cob. Also, there is a negative correlation between weights of all seeds in the ear with seed/cob rate, which means that by reducing the amount of grain weight, the rate of seed/cob increases. The results also showed that there is a negative correlation between the outer diameter ear and the rate of seed/cob, as well as the outer diameter ear with the number of leaves.

There was also a positive correlation between the number of rows in grain per ear and the rate of seed/cob. In total, there is a positive correlation between the number of rows and the number of seeds in the column and outer diameter ear with the weight of cob. (Table 1).

Pearson's liner correlation coefficients between traits

\begin{tabular}{|c|c|c|c|c|c|c|c|c|c|c|c|c|c|}
\hline & NR & NSC & WS & WC & SC & NOSC & HP & LE & OED & NON & SD & $\mathrm{LN}$ & WSW \\
\hline \multicolumn{14}{|l|}{ NR } \\
\hline NSC & 0.041 & & & & & & & & & & & & \\
\hline WS & -0.275 & 0.567 & & & & & & & & & & & \\
\hline WC & 0.53 & 0.452 & -0.292 & & & & & & & & & & \\
\hline $\mathrm{SC}$ & $0.505^{*}$ & 0.067 & $-0.743^{* *}$ & $0.832^{* *}$ & & & & & & & & & \\
\hline NOSC & $0.647^{*}$ & -0.135 & -0.072 & 0.201 & 0.2 & & & & & & & & \\
\hline HP & -0.413 & 0.093 & 0.076 & -0.032 & -0.019 & -0.114 & & & & & & & \\
\hline LE & 0.2 & 0.5 & 0.561 & -0.095 & -0.313 & 0.12 & -0.029 & & & & & & \\
\hline OED & -0.211 & -0.192 & 0.358 & 0.614 & $-0.616^{*}$ & -0.202 & -0.153 & 0.467 & & & & & \\
\hline NON & -0.368 & -0.361 & -0.091 & -0.67 & -0.401 & -0.485 & -0.004 & 0.206 & 0.435 & & & & \\
\hline $\mathrm{SD}$ & -0.484 & 0.071 & -0.088 & -0.249 & -0.041 & -0.395 & -0.288 & -0.197 & 0.026 & 0.3 & & & \\
\hline $\mathrm{LN}$ & -0.09 & 0.008 & -0.316 & 0.222 & 0.377 & 0.236 & 0.353 & -0.201 & $-0.646^{*}$ & -0.201 & 0.037 & & \\
\hline WSW & -0.023 & 0.14 & 0.523 & -0.24 & -0.502 & 0.097 & 0.1 & 0.069 & -0.243 & -0.061 & -0.371 & 0.137 & \\
\hline
\end{tabular}

number of rows (NR), number seeds on per cob (NSC), Weight of all seeds (WS), weight of cob (WC), seed/cob rate(SC), Number of seeds in column (NOSC), Length ear (LE), outer diameter ear (OED), number of nodes (NON), stem diameter (SD), weight 100 seeds in fresh (WSW), LE (length of ear), LN (leaf number). *,** and ns: Respectively, indicating a significant difference in the level of $1 \%, 5 \%$ and no significant difference

Plant height was positively and significantly associated with grain yield per plant. The association of plant height with all other traits was positive and significant. Similar result was also reported by Rahman et al, 2015 Ear height was positively and significantly associated with shelling percentage and fodder yield per plant. This was confirmed with the result of Vaghela et al, 2009. Correlation coefficients between the studied variables and total yield showed that only kernel no./row and 10 ear weight were significantly positively correlated with total yield under drought condition (Mostafavi et al, 2013).
Evaluation of Relationship between by GGE bi Plot

As you can see in Figure 1, there is a positive correlation between stem diameter, seed/cob rate and the number of nodes due to the low angle between them. Also, there is a positive correlation between the traits of cob weight, the outer diameter of the ear, the total seeds weight in the ear, due to the low angle. Also, there is a negative correlation between the weight of the ear and the number of nodes due to the large difference in the angle of the negative. There is also a negative correlation between the traits seed/cob rate and the outer diameter of the ear. 


\section{Scatter plot (Total - $100.00 \%)$}
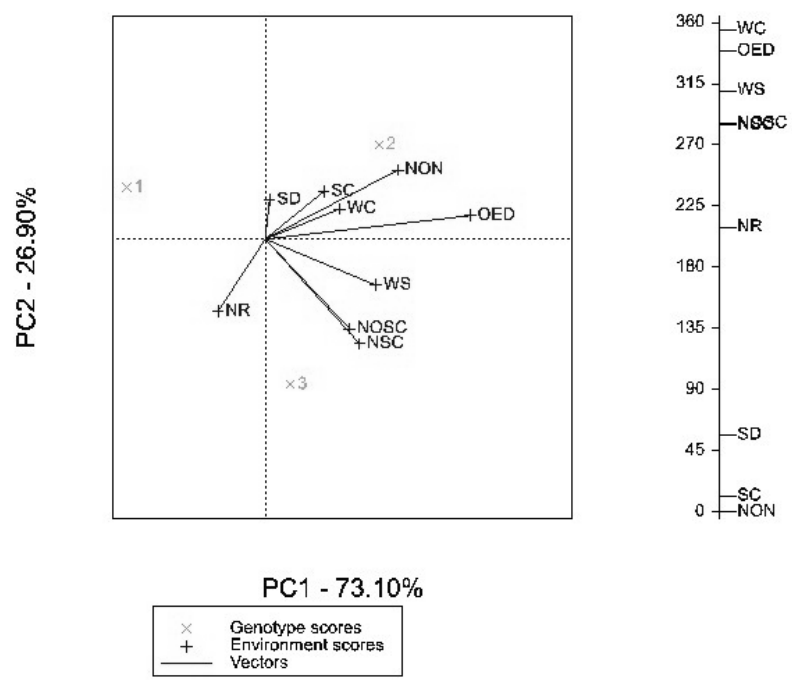

number of rows (NR), number seeds on per cob (NSC), Weight of all seeds (WS), weight of cob (WC), seed/cob rate (SC), Number of seeds in column (NOSC), Length ear (LE), outer diameter ear (OED), number of nodes (NON), stem diameter (SD), weight 100 seeds in fresh (WSW). G1: Armegnac, G2: Loupiac, G3: Sushi

\section{Identification of superior traits of yield using a graphical method GGE biplot}

The graph shows the ideal attribute. Ideal traits have the most recognition and representation capabilities among other traits. According to Figure 2, the traits of weight of all seeds and outer ear diameter had the highest effect on average yield and number of seeds per row showed the least effect on the average yield of hybrids. The mean of traits is as follows:

Outer ear diameter $<$ Weight of all seeds < number of nodes $<$ Number of seeds in column $<$ number seeds on per cob <weight of cob <seed/cob rate<stem diameter $<$ number of rows

\section{Determining the relationships between traits and Genotypes using the GGE biplot method}

In bi-plot Figure 3, a polygonal shape is created that connects the farthest genotypes (from the source of the biplot) to each other and provides comprehensive and detailed information. In this figure, we can see a triangle in which each angle represents each genotype. There are three traits inside the triangle. Stem diameter, the weight of cob, seed/cob rate are traits inside the triangle. These are important traits for each genotype in this study. Based on the number of nodes near the Loupiac hybrid, it can be stated that the Loupiac hybrid is the best genotype for the number of nodes. Also, based on the number of seed per cob near the Sushi hybrid, it can be concluded that the Sushi hybrid is the best genotype in terms of the number seeds per cob.
Figure 2: Rank the traits based on the ideal trait

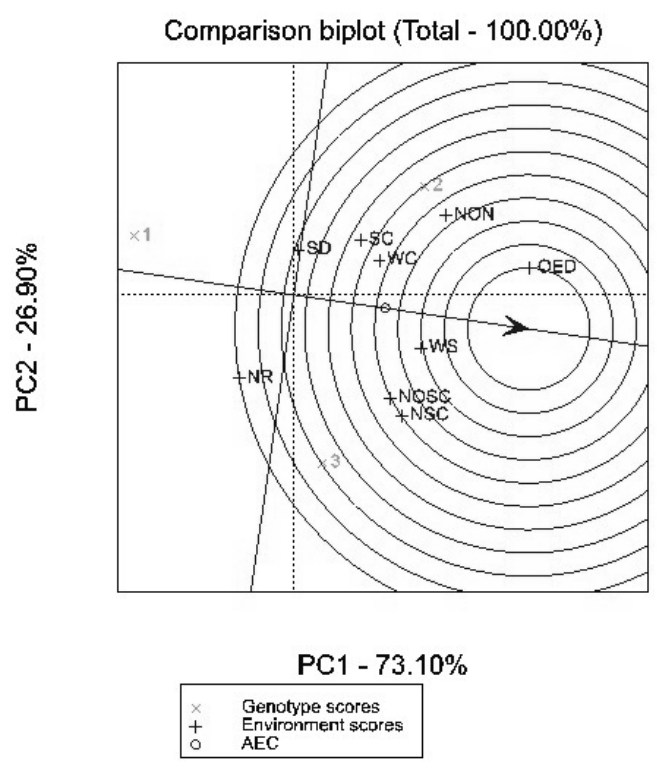

number of rows (NR), number seeds on per cob (NSC), Weight of all seeds (WS), weight of cob (WC), seed/cob rate (SC), Number of seeds in column (NOSC), height plant (HP), Length ear (LE), outer diameter ear (OED), number of nodes (NON), stem diameter (SD), leaf number (LN), weight 100 seeds in fresg (WSW). G1: Armegnac, G2: Loupiac, G3: Sushi) 
Comparing yield components of seven cultivars of maize in the Khoy region showed that there was a significant difference between yield and yield components of cultivars at the level of one per cent and the SC647 cultivar had the highest yield (Khalili Mahale et al., 2010).

\section{Figure 3: Bi plot for interaction between traits and genotype}
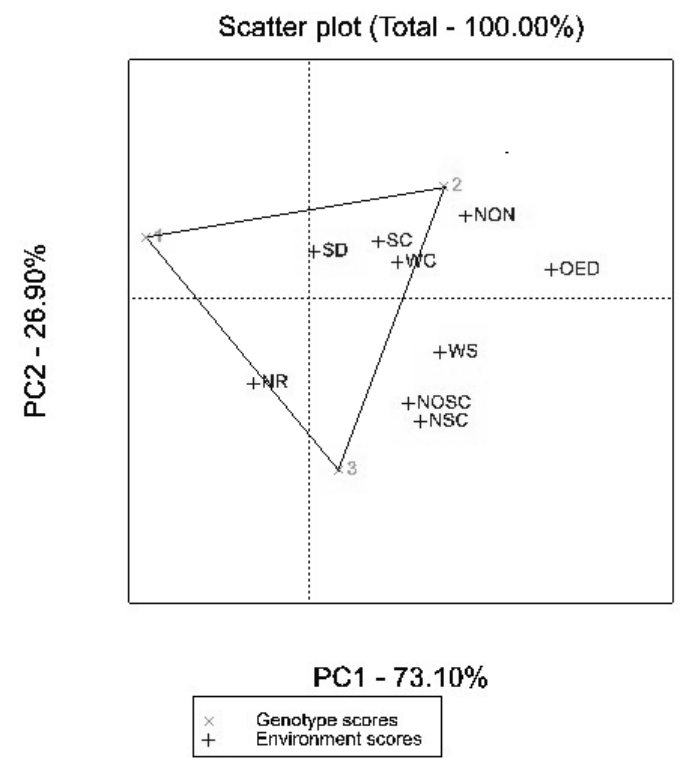

number of rows (NR), number seeds on per cob (NSC), Weight of all seeds(WS), weight of cob (WC), seed/cob rate(SC), Number of seeds in column(NOSC), height plant(HP), Length ear(LE), outer diameter ear (OED), number of nodes (NON), stem diameter(SD), leaf number(LN), weight 100 seeds in fresh(WSW). G1: Armegnac, G2: Loupiac, G3: Sushi

\section{CONCLUSION}

In this study, we evaluated three genotypes of agronomical traits by correlation analysis and GGe biplot too. we obtained positive correlation in seed/cob rate with weight of cob having the highest effect and negative correlation seed/cob maize with the weight of seed in an ear. The best hybrid was Loupiac in terms of yield and agronomical traits in Debrecen. The analysis of variances indicated significant differences among genotypes for all traits. For evaluation of cultivars with genetic potential, the GGE bi-plot graphical method was used. The measured traits were grain yield per plot, spike weight, 1000 kernel weight, fertile tiller number, spike length, awn length, peduncle length and plant height. General and specific combining ability for all traits were significant. Gascogne was the best general combiner of the Gaspard, the Ghods and the Pishtaz which had the highest SCA. These results were confirmed by the biplot analysis. Alvand cultivar was the best tester for 1000 kernel weight. Gaspard and Sardari cultivars were the best testers for grain yield (Mostafavi et al., 2013). In 2010, Mostafavi et al., stated that, in terms of genetic diversity and comparison of canola cultivars and the effect of genotype and traits, there is a significant difference between rapeseed cultivars for all traits. In this study, the ranking of genotype based on the trait showed genotype No. 3 was the best candidate and genotype10 as the most unpopulated variety for oil percentage.

\section{REFERENCES}

Ashofteh Beiragi, M.-Khavari Khorasani, S.-Sarmad Nabavi, M.Nikzad, F.-Zandipour, E. (2011): Study yield stability of commercial maize hybrids (Zea mays L.) evaluated in two planting dates in Iran. African Journal of Agricultural Research Vol, 6 (13): $3161-3166$

Chockan, R. (2010): Genotype, environment and genotype $x$ environment interaction effects on the performance of maize (Zea mays L.) inbred lines. Crop Breeding Journal ,1(2): 97-103.

Chockan, R. (2012): Maize and its characteristics. Agricultural Education Publishing.421 p.

Dehghani, H.-Dvorak, J.-Sabaghnia, N. (2012): Biplot analysis of salinity related traits in beard wheat (Triticum aestivum L.) Annals Biological Research 3:3723-3731.

Fan, X. M.-Kang, H. Y.-Zhang, J.-Tan C. (2007): Yield stability of maize hybrids evaluated in multi-environment trials in Yunnan, China. Agron. J., 99: 220-228. DOI: 10.2134/agronj2006.0144.

Kaplan, M.-Kokten, K.-Akcura, M. (2017): Assessment of Genotype $\times$ Trait $\times$ Environment interactions of silage maize genotypes through GGE Biplot. CHILEAN JOURNAL OF AGRICULTURAL RESEARCH 77(3) JULY-SEPTEMBER.

Kang, M. S.-Aggarwal, V. D.-Chirwa, R. M. (2006): Adaptability and stability of bean cultivars as determined via yield stability statistic and GGE biplot analysis. J. Crop Improve., 15: 97-120. http://www.informaworld.com/smpp/content content.

Morris, C. F.-Campbell, K. G.-King, G.E. (2004): Characterization of the end-use quality of soft wheat cultivars from the eastern and western U.S. germplasm 'pools.' Plant Genet. Res. 2:59-69.

Mostafavi, K.-Firoozi, M.-Mousavi, S. M. N. (2013): Effect of drought stress on yield and yield components of maize hybrids. Scientific Research and Essays, 8(24), pp.1145-1149.

Mostafavi, K.-Zabet, M. (2013): Genetic study of yield and some agronomic traits in bread wheat using biplot of diallel data. seed and plant improvement journal. number 3; page(s) 503 to 518 .

Rahman W, A. I.-Talukder, A. A.-Mamun, A.-Hanif. J. (2015): Studies on genetic variability, correlation and path analysis of yield attributes in maize genotypes (Zea mays L.). Journal of Chemical, Biological and Physical Sciences., 5 (4): 3964-3971.

Sabaghnia, N.-Dehghani, H.-Sabaghpour, S. H. (2006): Nonparametric methods for interpreting Genotype $\times$ Environment interaction of lentil genotypes. Crop Sci., 46: 11001106.http://crop.scijournals.org/cgi/content/abstract/46/3/110.

Samonte, S. O. P. B.-Wilson, L. T.-Mc Clung, A. M.-Medley, J. C. (2005): Targeting cultivars onto rice growing environments using AMMI and SREG GGE biplot analyses. Crop Sci., 45: 2414-2424. DOI: 10.2135/cropsci2004.0627. 
Schwietzke, S.-Kim, Y.-Ximenes, E.-Mosier, N.-Ladisch, M (2009): Ethanol production from maize. In Molecular Genetic Approaches to Maize Improvement (pp. 347-364). Springer, Berlin, Heidelberg.

Setimela, P. S.-Vivek, B.-Banziger, M.-Crossa, J.-Maideni, F (2007): Evaluation of early to medium maturing open pollinated maize varieties in SADC region using GGE biplot based on the SREG model. Field Crops Res., 103: 161-169. DOI: 10.1016/j.fcr.2007.05.010.

Vaghela, P. K.-Patel, D. B. D.-Parmar, J.-Macwana, S. S. (2009): Correlation and path coefficient analysis in baby maize (Zea mays L.). Research on Crops., 10 (1): 135-137.
Yan, W.-Rajcan, I. (2002): Biplot evaluation of test sites and trait relations of soybean in Ontario. Crop Science. 42: 11-20.

Yan, W.-Hunt, L. A.-Sheng, Q.-Szlavnics, Z. (2000): Cultivar evaluation and mega- environment investigation based on the GGE biplot. Crop Sci., 40: 597-605. http://crop.scijournals.org/cgi/content/abstract/40/3/597. 
